\title{
Alpha radiation induced space charge stability effects in semi-insulating silicon carbide semiconductors compared to diamond. ${ }^{\text {th }}$.
}

\author{
M. Hodgson ${ }^{\mathrm{a}, *}$, A. Lohstroh ${ }^{\mathrm{a}}$, P. Sellin ${ }^{\mathrm{a}}$ \\ ${ }^{a}$ Department of Physics, University of Surrey, Guildford, GU2 7XH, United Kingdom
}

\section{Abstract}

Although the use of semi-insulating silicon carbide material for radiation detection purposes has been previously demonstrated, its use in practical applications has been inhibited by space charge stability issues caused be defect concentrations within the material, the so called polarisation effect, by which the count rate and resultant spectrum changes with irradiation time.

This is a result of the charge carriers generated during irradiation filling deep level defects within the material, causing space charge build up and deactiving that trap level until the trapped charge is re-emitted. Consequently, the time dependence of the polarisation effect has been determined by a combination of parameters that can be influenced during operation, namely the incident radiation intensity, ambient light, temperature and bias. The material properties have also been considered through the use of materials with different defect capture cross sections, concentrations and energy level.

A thorough characterisation of the alpha irradiation induced polarisation phenomenon in semi-insulating silicon carbide has been conducted to demonstrate that stable operation detectors are in fact possible with this material. The effects were compared to single crystal diamond and polycrystalline diamond, which are known to exhibit similar polarisation issues.

The polarisation rate as an effect of incident flux, bias and temperature was determined, with the depolarisation rate as a function of ambient light

(C)British Crown Owned Copyright 2017/AWE.

*Corresponding author

Email address: michael.hodgson@becq.co.uk (M. Hodgson) 
and bias also demonstrated. Consequently it has been shown that stable operation can be maintained for detectors made from semi-insulating $\mathrm{SiC}$ material of active thickness $350 \mu \mathrm{m}$ at incident alpha radiation fluxes of $<0.7$ alphas per second per $\mathrm{mm}^{2}$ with high operating biases $(> \pm 400 \mathrm{~V})$. Furthermore, polarisation can be suitably managed or reduced through the use of light illumination and elevated temperatures (373K).

Keywords:

Semi-Insulating Silicon Carbide, Single Crystal Diamond, Polycrystalline Diamond, Irradiation stability, Polarization, Temperature dependance.

\section{Introduction}

The use of silicon carbide $(\mathrm{SiC})$ semiconductor material has been on the increase since the 1990's following a drive for electronics which could withstand high temperatures and radiation doses. As such high quality, low defect material is now commercially available for applications such as high-voltage electronics, ultra-violet photodiodes and light emitting diodes.

In addition to this, a lot of work has been conducted to investigate the performance of this material in detecting radiation [1][2][3][4][5]. This work has predominantly been conducted on thin epitaxial detectors, but work has also demonstrated that semi-insulating $\mathrm{SiC}$ (SiC-SI) materials have favourable particle and neutron detection properties [1][6][7][8][9] providing a potentially thicker and more cost effective detection alternative.

However, at the time of writing most semi-insulating $\mathrm{SiC}$ still suffers from relatively low charge collection efficiencies $(<50 \%)[9][10]$ and issues with stability, namely the polarisation effect by which the count rate and resultant spectrum changes with irradiation time [6][7][9]. These issues are primarily a result of high defect concentrations within the material.

A study has been conducted to fully characterise the polarisation effect within semi-insulating $\mathrm{SiC}$ radiation detectors and demonstrate methods to manage it. These management methods allow for the stable use of semiinsulating $\mathrm{SiC}$ material in practical radiation detection applications. As a benchmark, similar studies were carried out on single crystal diamond (D-SC) and polycrystalline diamond (D-PC), both of which have well documented polarisation phenomena for radiation detection applications [11][12][13][14]. 


\section{Theory}

The so called polarisation effect in semiconductor detectors made from wide band gap material is a phenomenon by which the spectrum and/or count rate varies and typically reduces during the irradiation [15]. It is generally accepted that polarisation occurs when trapped charge carriers remain immobilised for relatively long periods of time, such that the rate of charge trapping is greater than the rate of detrapping [15], although it is worth mentioning that polarisation occurs in thallium bromide $(\mathrm{TlBr})$ semiconductor detectors due to the physical movement of ions in the material when a bias is applied [16][17].

Charge carrier traps are a result of growth defects or impurities introduced into the material during growth, fabrication and/or operation. They act to capture created charge (electrons or holes) and immobilise them for a period of time or even neutralise them completely. Lost or delayed charge carriers result in a reduced signal pulse due to incomplete charge induction on the electrodes during the integration time of the system. Furthermore, the emissions of detrapped electrons and holes outside of the integration time of their associated event, may also add to the noise of the system [18].

A further consequence of trapped charge carriers is the creation of an ionised centre with a localised space charge region around it (Figure 1). While the rate of trapping is greater than the rate of detrapping, there will be a resulting buildup of trapped charge carriers and the space charge region will change throughout the material.

For localised charge carrier generation, by which the charge creation and subsequent trapping predominantly occurs within a localised region (e.g. alpha particle irradiation, where charge carrier generation is concentrated near the irradiated surface due to the short range of alpha particles in the material), the space charge build up can create a localised reduction of the electric field seen by the carriers, thus reducing the local charge drift velocity, leading to an increased probability of trapping and hence reduced resultant pulse height (Figure 1).

Uniform trapping occurs when charge carrier creation occurs throughout the material (e.g. gamma ray irradiation) resulting in a diluted build-up of space charge throughout the detector. Subsequently, subject to the number of traps present, stable operation can be achieved following an initial period of variation as all the traps are filled. However, a stable detector may not necessarily yield optimum counting efficiencies or resolutions and may also 
Figure 1: Concept of signal creation and localised polarisation in semiconductors.
(a) Electron-hole creation and migration in a biased semiconductor.

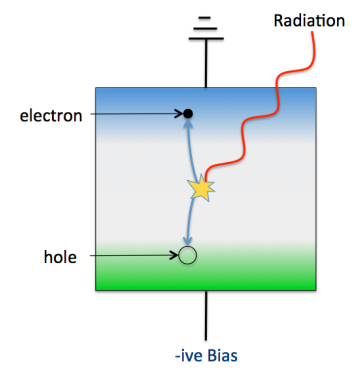

(b) Trapping of electron in deep level trap, with subsequent reduction in signal.

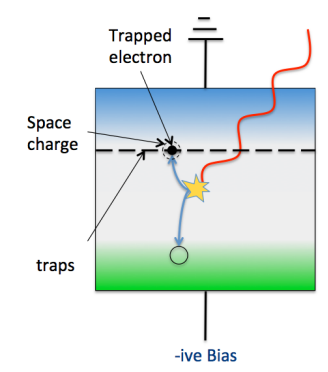

(c) Change in space charge region due to large concentration of trapped electrons.

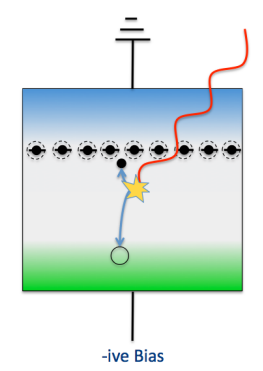

require increased applied electric field to compensate for the space charge build up.

The severity of these effects is subject to the type, concentration and location of the traps, as well as the primary charge carrier. These traps exist at specific energy levels within the band gap region, the energy of which the trapped charge carriers must subsequently overcome in order to once again freely move through the material. As given by Lutz [18], the average emission time of a trapped charged carrier, or the detrapping time, $\left(t_{t}\right)$ is dependent upon both the temperature $(T)$ and the energetic location of the trap within a specific material's bandage,

$$
t_{t}=\frac{1}{\sigma_{c} \cdot v_{t h} \cdot n_{i} \cdot e^{\frac{E_{i}-E_{t}}{k T}}}
$$

where $\sigma_{c}$ is the capture cross-section, $v_{t h}$ is the thermal velocity of the charge carriers, $n_{i}$ is the intrinsic carrier concentration and $k$ is the Boltzmann constant. $E_{i}$ and $E_{t}$ are the intrinsic (or Fermi) level and defect energy level respectively.

Therefore shallow traps (typically $<0.4 \mathrm{eV}$ ) are quite close to the allowed energy bands and charge carriers tend to easily migrate between the energy levels quickly. However, deep traps tend to exist near the mid-point of the forbidden region and as such the amount of energy required for the trapped carriers to migrate back to the allowed energy band may be large. 
A direct consequence of wide band gap material detectors is that the deep level trap energy value can be relatively large (typically $>0.7 \mathrm{eV}$ ) and subsequently thermal excitation of the trapped charge carrier is unlikely, leading to a long detrapping time. For example, in diamond traps within the band gap have been identified at $1.23 \mathrm{eV}$ [19] and $1.86 \mathrm{eV}$ [20] leading to an estimated room temperature detrapping time of $\approx 76$ days and $\approx 2 \times 10^{9}$ years respectively, where as the main defects in $\mathrm{SiC}$ are around $0.63 \mathrm{eV}$ and $0.97 \mathrm{eV}[21]$ and can last several hours, as shown in Table 1.

A further consequence of wide band gap material is that even the shallow traps can result in long periods of trapping relative to the integration time of the system (as shown in Table 1) resulting in transient stability issues. As such trap induced polarisation is quite prevalent in wide band gap materials such as diamond (D) [22], SiC [23], CZT [24] (Cadmium Zinc Teluride) and CdTe [25] (Cadmium Teluride).

An indication of the concentration of traps within a material can be gained from the mobility lifetime $(\mu \tau)$ product for each respective charge carrier, which is a combination of the charge carrier drift mobility $(\mu)$ within the material and the mean carrier lifetime $(\tau)$, that being the average period of time the created carriers exist and hence can travel before they are trapped, assuming free charge carrier recombination is negligible. This characteristic value defines the mean drift length $(\lambda)$ of the charge carriers through a material for a given electric field $(E)[15]$

$$
\begin{aligned}
\lambda_{e} & =\mu_{e} \tau_{e} E \\
\lambda_{h} & =\mu_{h} \tau_{h} E
\end{aligned}
$$

such that $\lambda$ needs to be greater than the sensitive region of the detector so that the charge carriers can travel the width of the detector and maximise the contribution to the signal output. Therefore when $\mu \tau$ is small it is more likely that charge carriers will be trapped.

Clearly one way to address polarisation effects within the material is to improve the growth, processing and fabrication techniques of the detectors in order to minimise the concentration of defects and impurities, which is by no means a simple task. However, there are several demonstrated methods to manage the polarisation in semiconductor detectors in order to make them suitable for practical detection applications.

\section{Thin Layer (Epitaxial)}

Certainly for $\mathrm{SiC}$ detectors, thin epitaxial layers (up to $100 \mu \mathrm{m}$ ) have been 


\begin{tabular}{|c|c|c|c|c|c|c|}
\hline Material & Trap & Type & Energy Location (eV) & $\sigma\left(\mathrm{cm}^{2}\right)$ & $t_{t}$ & Reference \\
\hline \multirow[t]{8}{*}{$4 \mathrm{H}-\mathrm{SiC}$} & \multirow[t]{3}{*}{$\mathrm{Cr}$} & \multirow[t]{3}{*}{ Impurity } & 0.15 & $\approx 1 \times 10^{-15}$ & $\approx \mathrm{ns}$ & {$[21][26]$} \\
\hline & & & 0.18 & $\approx 1 \times 10^{-15}$ & $\approx \mathrm{ns}$ & {$[21][26]$} \\
\hline & & & 0.74 & $\approx 2 \times 10^{-15}$ & $\approx \mathrm{S}$ & {$[21][26]$} \\
\hline & \multirow[t]{2}{*}{$\mathrm{Ti}$} & \multirow[t]{2}{*}{ Impurity } & 0.12 & $\approx 5 \times 10^{-15}$ & $\approx \mathrm{ns}$ & {$[21][26]$} \\
\hline & & & 0.16 & $\approx 1 \times 10^{-15}$ & $\approx \mathrm{ns}$ & {$[21][26]$} \\
\hline & $\mathrm{V}$ & Impurity & $0.9-0.97$ & $\approx 1 \times 10^{-16}$ & $\approx 5 \mathrm{hr}$ & {$[21][26]$} \\
\hline & $\mathrm{RD}_{4}$ & Defect & 1.5 & $\approx 5 \times 10^{-14}$ & $\approx 10^{2}$ years & {$[26]$} \\
\hline & $\mathrm{Z} 1$ & Defect & $0.63-0.65$ & $\approx 1 \times 10^{-14}$ & $\approx \mathrm{ms}$ & {$[21][26]$} \\
\hline \multirow[t]{6}{*}{ D-SC } & $\mathrm{B}$ & Impurity & 0.38 & $3 \times 10^{-16}$ & $\approx \mathrm{ms}$ & {$[27]$} \\
\hline & $\mathrm{N}$ & Impurity & 1.86 & - & $\approx 2 \times 10^{9}$ year & {$[20]$} \\
\hline & & Defect & 0.31 & $\approx 3 \times 10^{-16}$ & $0.5 \mathrm{~ms}$ & {$[28]$} \\
\hline & - & Defect & 0.39 & $3.2 \times 10^{-19}$ & $13 \mathrm{~ms}$ & [19] \\
\hline & - & Defect & 1.14 & $9.5 \times 10^{-14}$ & $\approx 76$ days & [19] \\
\hline & - & Defect & 1.23 & $4 \times 10^{-13}$ & $\approx 13 \mathrm{hr}$ & [19] \\
\hline
\end{tabular}

Table 1: Properties of deep level traps in $4 \mathrm{H}-\mathrm{SiC}$ and CVD diamond. $\sigma$ is the capture cross-section for charge carriers and $t_{t}$ is the detrapping time at $300 \mathrm{~K}$. 
shown to have no polarisation effects during operation [29][30][31]. This is in part due to better growth techniques for the smaller volumes of material, as well as the fact that less volume equates to physically fewer defects and impurities. Siffert et al. [25] also suggest that the ease by which a thinner layer of material can be fully depleted also plays a role, as it ensures the charge collection efficiency remains essentially constant even if all the deep level traps were filled.

For bulk material this would however require improvements at growth level which is currently not yet available through existing commercial material supply.

\section{Contacts}

Choosing a suitable contact material for wide band gap material detectors has been shown to reduce and even stop polarisation effects [32][33]. The injection of charge carriers opposite to those trapped by deep levels (e.g. inject holes for trapped electrons) quickly neutralises the immobile carriers before the build up of space charge alters the sensitive detection region. However, the injection of carriers leads to an increase in leakage current within the detector [34] and as such is only suitable for high resistivity materials [32].

Therefore, it has been shown in CdTe that Schottky barrier detectors tend to polarise the detector quickly [32] as the Schottky space charge barrier inhibits the flow of current and reduces the chance of charge injection and hence neutralisation. Similarly, ohmic detectors have been shown to reduce the polarisation effects within CdTe [32] due to an increased chance of neutralisation through flowing electrons from the contact [32].

It is worth noting that the process of contact fabrication can result in significant radiation doses to the material that may in itself result in deep level trap filling, thus improving the long term stability of the device through the process rather than the contact itself [35].

In a similar sense to the point above, contact design would need to be implemented at the fabrication phase and may well be impractical in the short term for those looking to use the material in practical applications.

\section{Bias}

Whereas the effect of applying a bias while under irradiation starts polarisation within the detector [25], the effect of removing the bias has been shown to depolarise (recover) wide band gap semiconductor detector performance. The process is primarily a result of carrier recapture and subsequent neutral- 
isation, rather than detrapping [34]. This is due to liberated charge carriers migrating locally or slowly through the material, rather than being quickly separated and collected by the electrodes when under bias, thus increasing the chance of recombination. The rate of depolarisation is therefore relatively quick compared to polarisation, however the material will re-polarise once biased and irradiated again as the traps will still exist.

Full depolarisation within $\mathrm{SiC}$ has been suggested to take in the order of 10 minutes [23], where as in $\mathrm{D}$ it can be in the order of hours from Table 1. Clearly this is not suitable for regular sustained detection, however extended periods of operation have been demonstrated in other wide band gap materials by regularly pulsing the bias supply between operational level and $0 \mathrm{~V}[22][32][36][37][38]$. The process effectively creates a semi-polarised state where trapping and neutralisation are in equilibrium.

The disadvantage to this method is a more complex power supply circuit and the potential of electromagnetic interference on the signal amplification circuit caused by the switching bias.

A further alternative is to increase the bias as the trapping increases. Effectively this increases the electric field sufficiently so that the created charge carriers can be collected at the electrode. Essentially this technique has been demonstrated in $\mathrm{D}$ as part of the priming process previously mentioned, but it is limited by the carrier saturation velocity.

\section{Light Illumination}

On both CdTe [39] and Diamond [22][40] work has been conducted which shows that illumination with visible or near-visible wavelength light can depolarise the material and even improve the subsequent rate of polarisation (i.e. the rate at which the detector performance changes). It has been proposed that this occurs when the light provides sufficient energy to depopulate traps in the device.

Once again it should be noted that the unpolarised detector is temporary as the deep level traps will still be present within the material, thus polarisation will again occur when the material is biased and irradiated. However, it is possible that continual light pulsing could potentially create an equilibrium state between trapping and neutralisation, leading to stable operation.

It should also be noted that the wavelength of the incident light needs to be carefully selected, as it can induce polarisation in the detector rather than depolarise it. The work of Sato et al. [39] on CdTe showed that at visible photon energies greater than the material's band gap, the detector can fully 
depolarise, where as at energies equal to or lower than the band gap the detector can fully or partially polarise respectively. Similarly Manfredotti et al. [41] have shown in chemical vapour deposition (CVD) diamond that illumination with blue light $(450 \mathrm{~nm})$ before or during radiological irradiation results in a stable detector.

\section{Priming}

Within some wide band gap semiconductor materials (particularly Diamond) priming is often employed as a way of stabilizing the polarisation effect within the material [11][12][42][43]. This process involves irradiating the detector to high doses (usually in the region or 5-20Gy[43]) in order to saturate the detector with a large electron-hole concentration, a significant proportion of which are then captured by the deep level traps. As all the traps are filled, electron-hole pairs created as a result of subsequent irradiations are less likely to be trapped within the material, increasing lifetime of the carriers for that irradiation.

By applying a higher bias, the created charge carriers can overcome any space charge variation and be fully collected at the electrodes. Furthermore, as the trapped charges are immobile for relatively long periods of time (provided the carriers are not detrapped through heating, light etc) the response remains very stable over reasonable periods of time [12]. This method of controlling polarisation is inconvenient for many applications as it requires significant doses to the detector and only solves the issues associated with deep level traps, with shallow trap polarisation effects still being present.

\section{Temperature}

An increase in temperature has been shown to increase the rate of polarisation within some wide band gap semiconductor materials [25][39][44]. Similarly lower, $<0^{\circ} \mathrm{C}$ temperatures have been shown to decrease the rate of polarisation [25] by reducing thermal carrier generation and stabilising the polarisation effect by decreasing the detrapping time, as suggested in Equation 1.

However, increased temperatures can also lead to a reduction in the polarisation effect when the trapping rate is in equilibrium with the thermally induced detrapping rate, thus reducing any cumulative space charge build up. Therefore for wide band gap, low intrinsic carrier concentration semiconductor materials that are capable of very high temperature operation, such as $\mathrm{D}$ and $\mathrm{SiC}$, it is feasible specific temperature values could lead to 
stable operation without resulting in a large thermally induced background noise.

In practical applications, manipulation of the operational temperatures for $\mathrm{SiC}$ and $\mathrm{D}$ detectors could potentially result in complicated cooling systems (peltier coolers, liquid nitrogen dewars, etc.) or increased noise from the circuitry components due to high temperatures. However, Guerrero et al. have demonstrated a stable D detector solution utilising discrete heating contacts for medical applications which opens up possibilities to other areas $[45]$.

\section{Experimental Methods}

The single crystal diamond (D-SC) and polycrystalline diamond (D-PC) detectors were fabricated from Element Six and Diamond Detectors Limited material respectively, as described by [9][46][47]. The D-SC was $500 \mu \mathrm{m}$ with $4 \mathrm{~mm} \times 4 \mathrm{~mm}$ sputter coated platinum contacts and was mounted on a ceramic board for testing. The D-PC was $300 \mu \mathrm{m}$ thick with $\varnothing 6.5 \mathrm{~mm}$ sputter coated gold metal contacts and was mounted on a printed circuit board.

Two semi-insulating silicon carbide ( $\mathrm{SiC}-\mathrm{SI}$ ) detectors were used for this testing, SiC-SI and SiC-SI-Cr5, both of which were fabricated from $\approx 350 \mu \mathrm{m}$ Cree $4 \mathrm{H}-\mathrm{SiC}$ material as discussed in [6][9][10]. Both detectors had sputter deposited $5 \times 5 \mathrm{~mm} \mathrm{Ti} / \mathrm{Pt} / \mathrm{Au}$ Schottky and Ni/Au ohmic contacts with appropriate annealing.

The SiC-SI and D-PC detectors were operated at $-400 \mathrm{~V}$, where as the D-SC was operated at -200V. Unless otherwise stated, the detectors were exposed to at least 15 minutes of room ambient light, while at $0 \mathrm{~V}$ bias, between each radiation exposure in order to partially de-polarise the detector by detrapping carriers from shallow traps, essentially "resetting" the detector, as will be presented later.

Measurements utilised ORTEC 142A charge sensitive preamplifiers; ORTEC 570 or 572 shaping amplifiers; an ORTEC 710 quad-bias supply; and ORTEC Easy-MCA with associated Maestro software. The detectors were either mounted in a vacuum cryostat or a bespoke, light sealed diecast metal test box with a radioisotope source exposure tray that allows for a source to be moved in front of or away from the detectors without exposing them to light. Exposures in the bespoke test box were in air at standard pressure.

Where light was required it was standard ambient room lighting, where as the test radiation sources were either a $3 \mathrm{kBq}, 60 \mathrm{kBq}$ or $185 \mathrm{kBq}$ Am-241 
alpha radiation emitter. For each radiation test the source was placed the same distance away $(\approx 11 \mathrm{~mm})$.

For temperature specific testing the detectors were mounted on ceramic boards which were connected to a Heat / Cool plate controlled with either an Oxford Instrument ITC 503 (Serial number E502/319) or Mercury ITC respectively respectively [6]. Where cooling was required the system was connected to a reservoir of liquid nitrogen.

Energy calibration of the detectors was conducted using pulser-capacitor calibrations as described by Siegbahn [48].

\section{Results}

From Figure 2 it is clear that the D-SC, D-PC and SiC-SI detectors all demonstrate alpha induced polarisation effects during irradiation, that being a change in the spectroscopic response (both peak position and count rate) with time. Within D-PC and SiC-SI this manifests itself as a steady movement of the alpha spectral response from higher channels to lower ones and potentially into the detector system's background noise.

Rather than steadily moving to lower channels, the D-SC alpha spectra produces two energy peaks, one at maximum $\left(P_{\max }\right)$ and one at $\approx 50 \%$ of maximum $\left(P_{\min }\right)$. During the initial stages of irradiation, the majority of counts exist in $P_{\max }$. As the irradiation continues, counts seem to transfer from $P_{\max }$ to $P_{\text {min }}$, up until the point by which $P_{\min }$ represents the main spectra and is stable, a characteristic also demonstrated by Souw and Meiluna [49] with a lower resolution detector.

This observation demonstrates the traps within the material being steadily filled with the created charge carriers until they reach saturation (i.e. the polarisation rate is in equilibrium with the de-trapping rate), after which a stable field, and therefore spectrum, is maintained.

The existence of these two spectrum 'states' during irradiation is a result of the relatively good mobility lifetime product $(\mu \tau)$ ensuring that the electrons and holes are capable of traversing a greater distance in the material while avoiding a significant amount of trapping, even with a localised space charge build up caused by the charge carriers that do get trapped. For D-SC the electron and hole mobility lifetime product $\left(\mu_{e} \tau_{e} / \mu_{h} \tau_{h}\right)$ has been determined to be $5.6 \pm 0.1 \times 10^{-5} / 6.1 \pm 0.3 \times 10^{-5} \mathrm{~cm}^{2} \mathrm{~V}^{-1}$ respectively [9], where as for D-PC and SiC-SI $\mu_{e} \tau_{e} / \mu_{h} \tau_{h}$ was a factor of 1000 and 10 less with values of $8.0 \pm 0.9 \times 10^{-8} / 6.7 \pm 0.7 \times 10^{-8} \mathrm{~cm}^{2} \mathrm{~V}^{-1}$ and $3.77 \pm 0.01 \times$ 


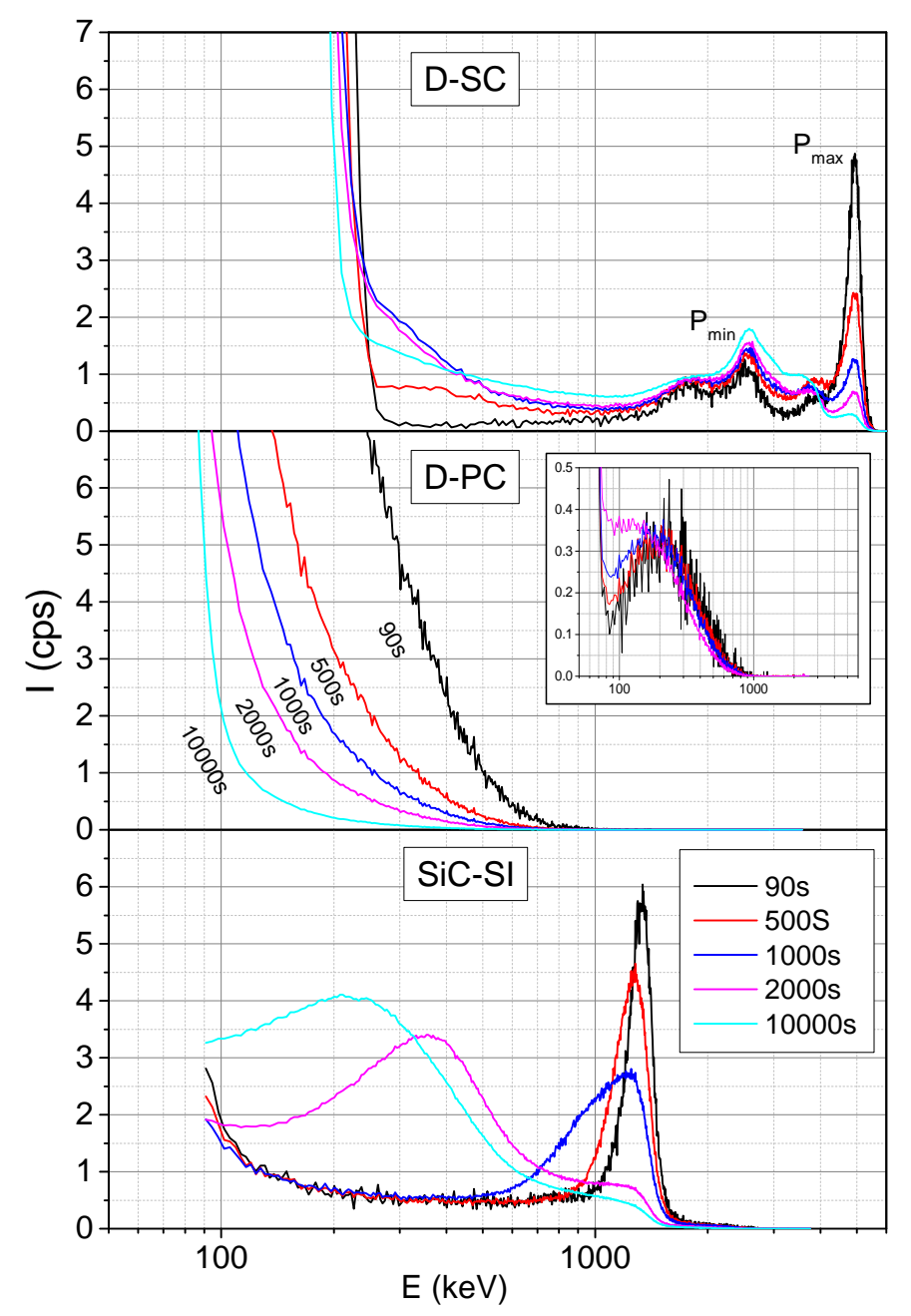

Figure 2: Count rate $(I)$ against energy $(E)$ for Am-241 alpha particles as a function of irradiation time. A $185 \mathrm{kBq}$ source was used for all tests with the exception of the D-PC inset graph where $3 \mathrm{kBq}$ was used. The D-SC $(-200 \mathrm{~V}, 524 \alpha / \mathrm{s})$, SiC-SI $(-400 \mathrm{~V}, 645 \alpha / \mathrm{s})$ and inset D-PC $(-400 \mathrm{~V}, 125 \alpha / \mathrm{s})$ graphs were taken in a $8 \times 10^{-2}$ mbar vacuum, where as the main D-PC graph was in air at standard pressure $(-400 \mathrm{~V}, 4384 \alpha / \mathrm{s}$. 
$10^{-6} / 0.34 \pm 0.01 \times 10^{-6} \mathrm{~cm}^{2} \mathrm{~V}^{-1}$ respectively [9] which is why the $P_{\min }$ point is below the noise edge.

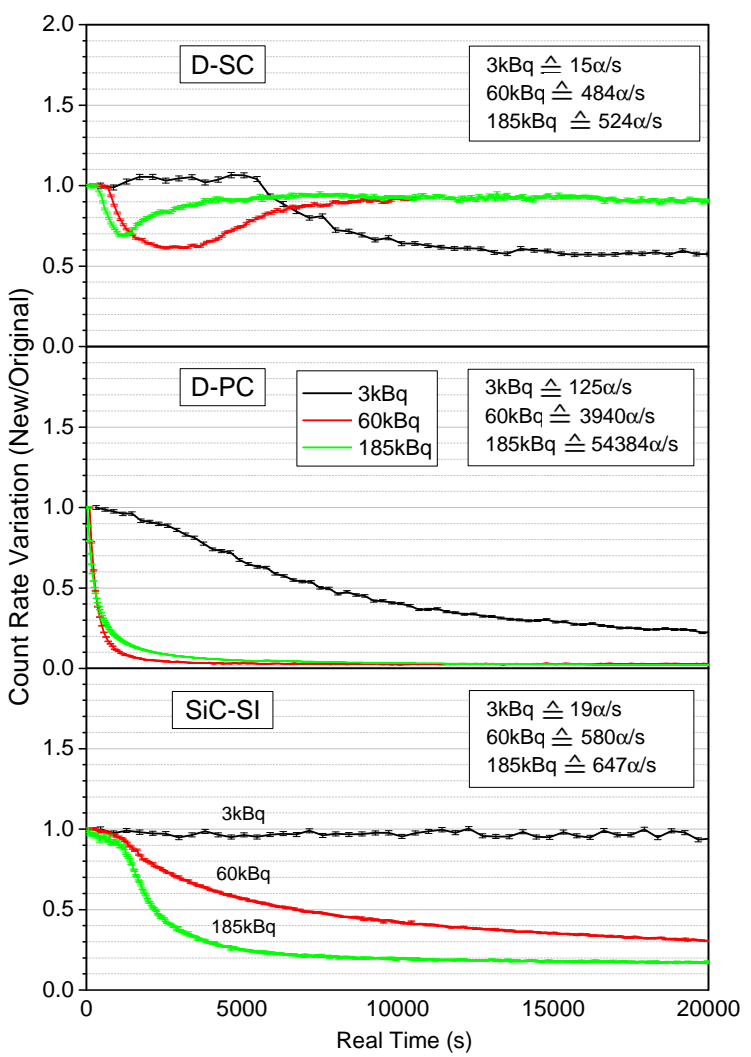

Figure 3: Count rate variation $\left(I / I_{0}\right)$ against time as a function of Am-241 alpha source activity in air. The specific flux incident upon the detector for each source activity has been stated. This takes into account the detectorto-source geometries and any losses. The flux for each activity was also calibrated with a known high precision detector. The detectors were at 200V (D-SC) or -400V (D-PC, SiC-SI).

Ideally the peak position within the spectrum gives the best indication of polarisation, as it is irrespective of the noise threshold. However, in several of the graphs presented the variation of the count rate is used as the determination of peak position was quite difficult for some of the D-PC tests (particularly when at standard pressure) and the error in the peak position 
was very large for all detectors when the peak position moved into lower energies, the variation in the count rate has been used.

The count rate is the total number of counts in the spectrum above a given noise threshold, divided by the time taken to acquire them and hence depends on the threshold level and the detection system's ability to effectively eliminate electronic noise. For this study, the threshold was generally selected to be 1 channel above the point where the background or noise spectrum was less than 1 count per second (cps). For most of the detectors, the main bulk of counts are in the alpha peak and therefore the count rate variation does represent a reasonable analogy of the peak position when close to the noise edge, as will be confirmed in Figures 6 and Figures 7 .

However, within Figure 3 this count rate-to-peak position correlation breaks down for the D-SC detector when the polarisation rate stabilises. Initially the D-SC demonstrates a decrease in count rate as traps are filled and contribute less to the detection signal due to the shorter distances travelled in the material. As the traps are filled, the charge carriers are able to fully traverse the material again and the count rate begins to increase and normalise, despite the peak position being at a lower (but stable position) due to the change of the space charge region.

Figure 3 also shows how the polarisation rate changes as a function of the incident alpha radiation flux. As might be expected, as the alpha flux increases (here represented by the emission rate of different Am-241 alpha sources) the rate of polarisation increases due to an increase in the number of charge carriers created and therefore trapped in shallow levels within the material. Conversely, as the polarisation rate increases the time to reach $80 \%$ of the original count rate $\left(t_{80}\right)$ decreases, as shown in Figure 4.

The decrease in $t_{80}$ for these detectors (and therefore increase in polarisation rate) can be described by Equation 4 which was estimated using a simple exponential decay function in Origin with a Levenberg Marquardt empirical iteration method.

$$
t_{80}\left[0<\phi<-1 / R_{0} \cdot \ln \left(y_{0} / A_{1}\right)\right]=A_{1} \cdot e^{-\phi / R_{0}}+y_{0}
$$

Here $\phi$ is the incident flux, where as $A_{1}, R_{0}$ and $y_{0}$ are fitting parameters characteristic of the material under test (shown in Table 2) for a given geometry and applied bias.

It is worth noting that Equation 4 and the values in Table 2 require more data points to predict the performance over different materials, geometries 


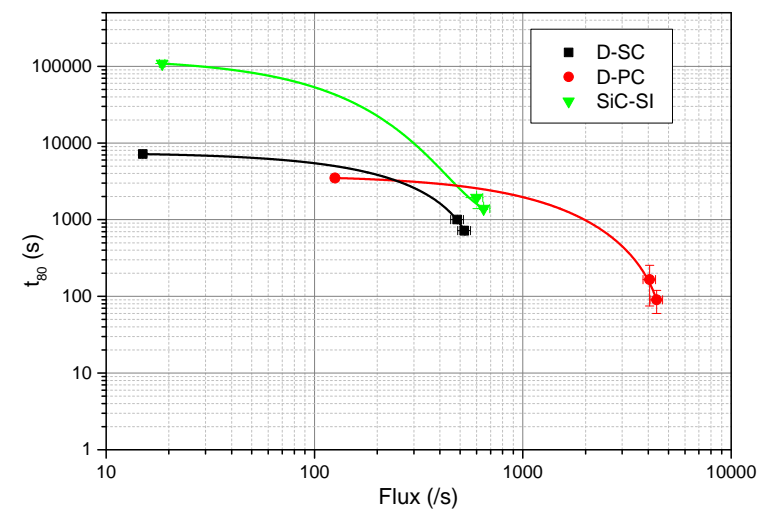

Figure 4: Time to reach $80 \%$ of the original count rate $\left(t_{80}\right)$ against Am-241 alpha flux from $3 \mathrm{kBq}, 60 \mathrm{kBq}$ and $185 \mathrm{kBq}$ sources in air. Plotted lines are fitted to Equation 4. A fixed source position was used for all irradiations.

\begin{tabular}{|c|c|c|c|}
\hline Detector & $\begin{array}{c}A_{1} \\
(\mathrm{~s})\end{array}$ & $\begin{array}{c}R_{0} \\
\left(\mathrm{~s}^{-1}\right)\end{array}$ & $\begin{array}{c}y_{0} \\
(\mathrm{~s})\end{array}$ \\
\hline $\mathrm{D}-\mathrm{SC}$ & 9266 & 397 & -1751 \\
$\mathrm{D}-\mathrm{PC}$ & 3973 & 1623 & -177 \\
SiC-SI & 126987 & 112 & -982 \\
\hline
\end{tabular}

Table 2: Fit parameters for Equation 4, defined by Origin fitting software. Detectors were tested at -200V (D-SC) or -400V (D-PC, SiC-SI). 
and biases, but the exponential decay function certainly gives a reasonable representation of the polarisation induced change in all the materials tested and is in very good agreement with the form suggested by Rogalla et al. [7]. Within Equation $4, R_{0}$ reflects the rate of polarisation and as such Table 2 suggests that $\mathrm{SiC}-\mathrm{SI}$ is the slowest polarising material. However, this does not take into consideration the count rate recovery in D-SC, clearly demonstrated in Figure 3, which would make D-SC the most stable of the polarising detectors over a long period.

Figure 4 does show that SiC-SI has a lower initial polarisation rate for low radiation fluxes relative to the diamond detectors, with SiC-SI providing a very stable count rate over a long period in Figure 3. This is a result of the de-trapping rate being greater than or equal to the charge carrier creation rate and would seem logical considering the main shallow traps have a longer detrapping time in $\mathrm{D}$ than in $\mathrm{SiC}$. Therefore, subject to the incident flux rate and the ionisation density (discussed in [6]) semi-insulting $\mathrm{SiC}$ can provide very stable radiation detection properties.

Furthermore, as the polarisation rate has demonstrated a relationship to both the energy levels of the main traps and the mobility lifetime product, in future work it may be interesting to see if Equation 4 and the associated material constants in Table 2 can be used to estimate these material characteristics. It is worth noting however, that the data presented is primarily for localised polarisation close to the electrodes due to shallow penetration depth of the alpha particles ( $\approx 17 \mu \mathrm{m}$ for $\mathrm{D}$ and $\approx 18 \mu \mathrm{m}$ for SiC [50]). Polarisation is expected to be more pronounced compared to scenarios where charge carriers are generated throughout the whole device thickness [51][52].

As has been discussed, localised trapping (as would be found with alpha radiation) results in a build up of space charge near the irradiated electrode that has the effect of reducing the electric field, thus inhibiting the movement of future created charge carriers and increasing the probability of being trapped themselves. As would be expected from Equations 2 and 3, increasing the applied electric field increases the mean drift length and gives the created charge carriers sufficient velocity both to overcome this space charge "barrier", therefore reducing the probability of being trapped. This has been demonstrated in Figure 5 where the rate of polarisation decreased as the bias is increased. The effectiveness of this technique will be limited by the saturation velocity of the charge carriers, a characteristic property of the material itself.

The rate of polarisation was larger (i.e. was quicker to polarise) for posi- 


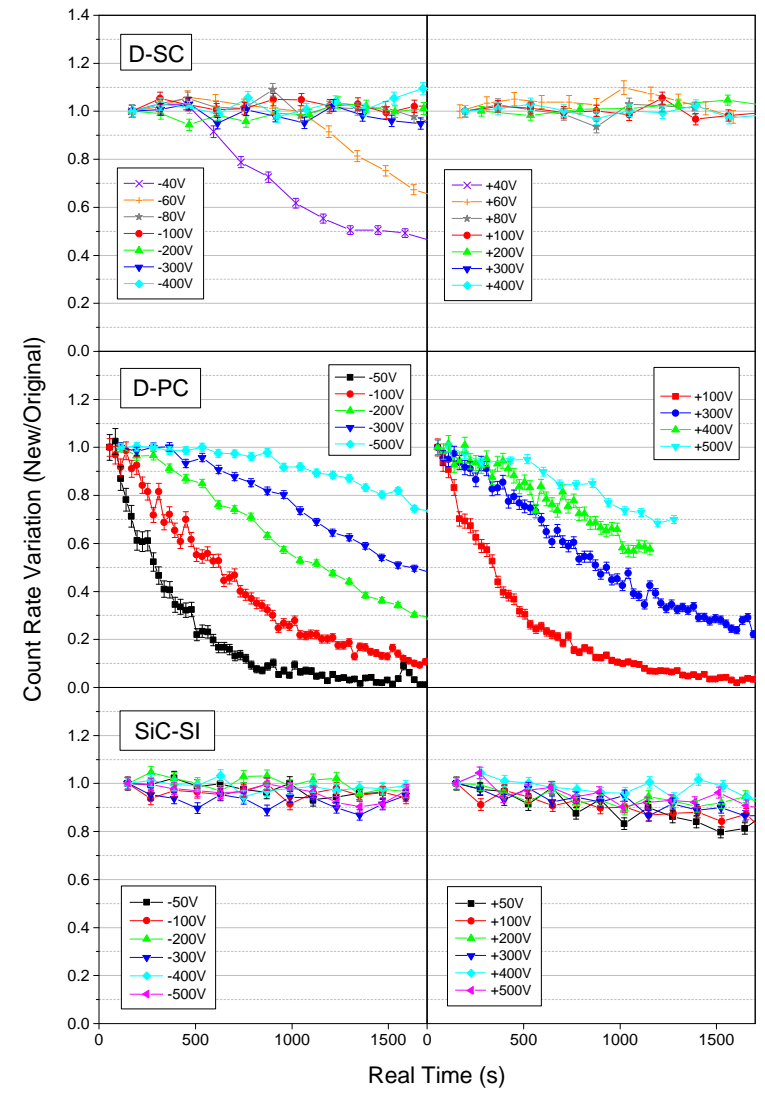

Figure 5: Count rate variation as a function of bias during irradiation with $3 \mathrm{kBq}$ Am-241 alpha particles in air. Flux: D-SC, $15 \alpha / \mathrm{s} ;$ D-PC, $125 \alpha / \mathrm{s}$; SiC-SI, $19 \alpha / \mathrm{s}$. 
tive bias in the D-PC and SiC-SI detectors when compared to negative bias which is a result of the lower mobility lifetime product for holes relative to electrons in each material [9]. Where as the D-SC the polarisation rate under negative bias, particularly at low fields strengths, was larger than positive bias, again a result of the higher hole mobility lifetime relative to electrons in this material [9].

From Figure 3 and Figure 5 it is clear that stable operation within polarising materials can be achieved when the irradiation setup allows for equilibrium between the trap/de-trap rate. Therefore techniques were investigated to improve stability by increasing the de-trapping or depolarisation rate. Decreasing the trapping rate would require solutions related to growth technique and/or doping, making it less viable route in the short term for operational detectors when designing practical applications.

The depolarisation rate of SiC-SI as a function of the applied bias and ambient light has been demonstrated for the count rate and centroid variation in Figures 6 and 7 respectively. Between each iteration the detectors were full depolarised (0V bias, >15mins light) between each iteration tested, so $1 \mathrm{hr}$ irradiation $\rightarrow$ depolarisation test $\rightarrow$ 1hr irradiation $\rightarrow$ full depolarisation. Therefore the figures highlight the repeatability of the polarisation effect.

Furthermore, these plots validate the correlation between count rate and centroid energy peak polarisation discussed earlier. There is more variation in the centroid energy peak from test-to-test which is due to the counting statistics in the main peak channel as a result of the timing periods used for the analysis (every 90s) and the fact that these tests were conducted in air in order to use the capabilities of the bespoke test box, consequently this results in a broader, lower intensity alpha peaks.

Figures 6 and 7 both show that removing applied bias (0V) and illuminating with light (No Bias, Light), depolarises the material following a 1 hour irradiation. The figures also demonstrate that simply removing the source (No Bias, No light) also leads to depolarisation. This results reiterates the notion that shallow traps are the key driver for the polarisation effect as the room temperature shallow level detrapping times are more in line with these results than the deep level detrapping times that can take several hours or even years. Extrapolation from data presented in Figure 7 would suggest a depolarisation time of around 30-60 minutes would be required for full depolarisation using the No Bias, No light technique in the SiC-SI detector.

The depolarisation rate as a function of bias is very small and is only really apparent when comparing the Bias, No light and No Bias, No light 


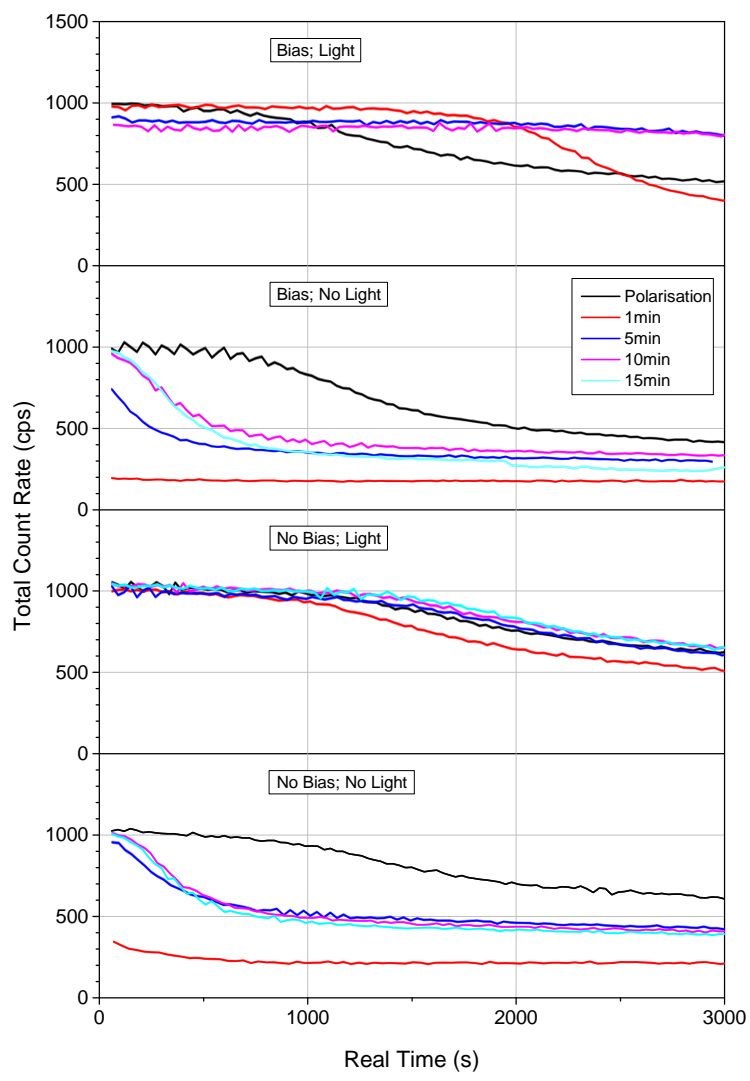

Figure 6: Total count rate above a given threshold against alpha irradiation time, as a function of depolarisation times for SiC-SI. Alpha source was Am241 in air $(60 \mathrm{kBq}, 587 \alpha / \mathrm{s})$. Bias was $-400 \mathrm{~V}$ and light was ambient room light. Polarisation represented $1 \mathrm{hr}$ irradiation at $-400 \mathrm{~V}$ in the dark with a fully recovered detector prior to any depolarisation test (i.e. $>15 \mathrm{mins}$ ambient light, at 0V). 


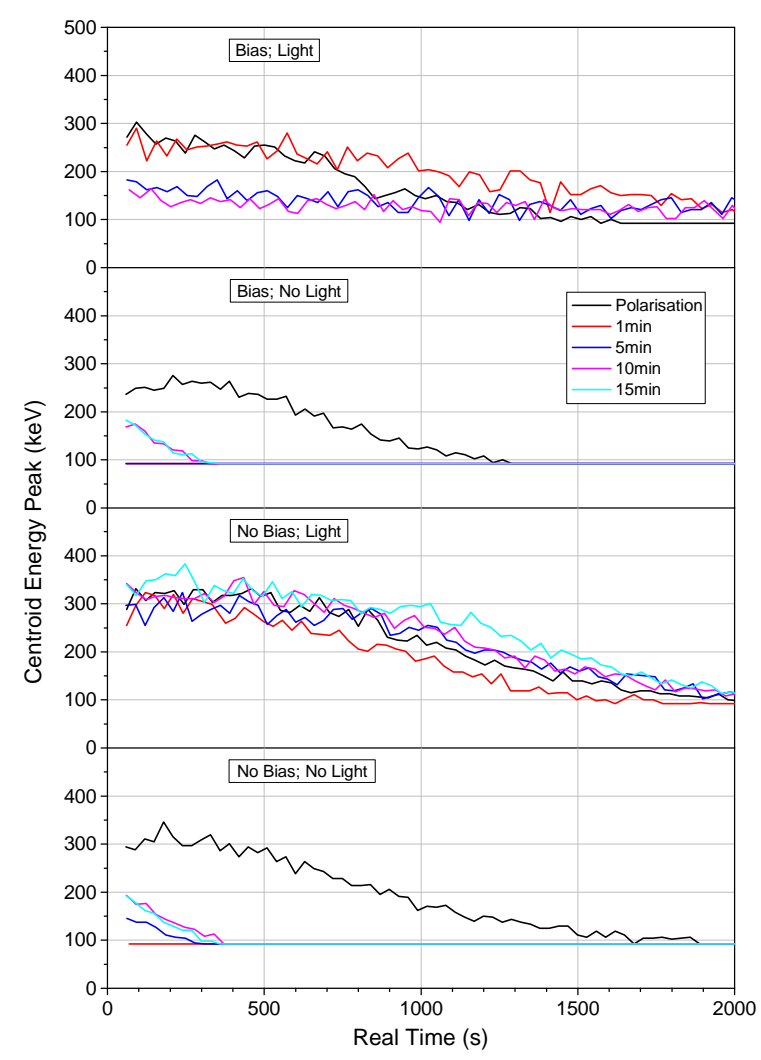

Figure 7: Energy peak centroid position against alpha irradiation time, as a function of depolarisation times for SiC-SI. Alpha source was Am-241 in air $(60 \mathrm{kBq}, 587 \alpha / \mathrm{s})$. Bias was $-400 \mathrm{~V}$ and light was ambient room light. Polarisation represented $1 \mathrm{hr}$ irradiation at $-400 \mathrm{~V}$ in the dark with a fully recovered detector prior to any depolarisation test (i.e. >15mins ambient light, at $0 \mathrm{~V})$. 
data. This method relies on detrapping and neutralisation as the key process for depolarisation, so essentially relatively long time periods and the probability of neutralisation from a contact injected charge carrier. The result is incomplete depolarisation (i.e. detector not returned to the original performance) for the time periods tested and subsequently quicker polarisation times following re-irradiation, highlighted in Figure 8.

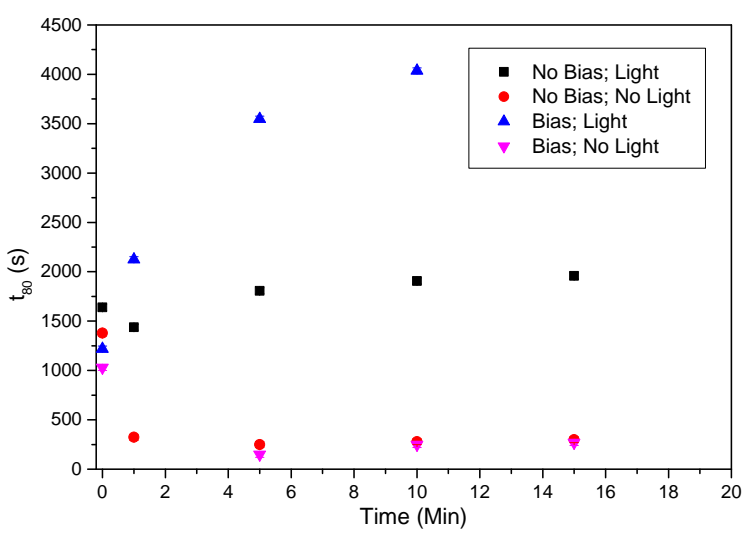

Figure 8: Time to reach $80 \%$ of the original count rate $\left(t_{80}\right)$ under irradiation of Am-241 in air $(60 \mathrm{kBq}, 587 \alpha / \mathrm{s})$ against depolarisation time for SiC-SI detector at $-400 \mathrm{~V}$.

By far the most effective method for depolarisation is illuminating with ambient light. For No bias, Light, the material fully recovers, even after only 1 minute, with good repeatability from test-to-test. This validates the full depolarised test procedure described (15mins with 0V bias and light illumination). However, Bias, Light leads to recovery with a lower polarisation rate following re-irradiation, i.e. the detector is more stable. In fact, an improvement in the polarisation rate occurs for both light illumination tests, as can be seen in Figure 8.

Recovery with light is well known in diamond [49] and other wide band gap materials like CdTe [39]. It is a result of the optical photons providing sufficient energy to liberate charge carriers from shallow traps (i.e. above 0.4 $\mathrm{eV}$ ), thus diluting the localised space charge build up and "resetting" the device.

When illuminated under 0V bias the shallow trapped charge carriers are liberated and as an electric field is not present, are free to recombine. The 
trap is then available for capturing further liberated electrons or holes when irradiated under bias once again. However, when the illumination occurs under bias, the existing charge carriers are again liberated, but additional optically stimulated charge carriers are also created (subject to the light intensity) which fill the available traps. Any further charge carriers created though radiation interactions are then free to migrate through the "optically primed" material without being trapped. The field present will of course be different to a fully unpolarised detector due to the trapped charge (i.e. transient space charge effects), but as $\mathrm{D}$ and $\mathrm{SiC}$ are both transparent to visible light, the trapping will be uniform and diluted relative to localised alpha radiation induced trapping [49].

Therefore for the Bias, Light data, stable operation is due to reduced trapping of radiation induced electron-hole pairs due to already filled traps. This is a particularly important feature for $\mathrm{SiC}$ as it is transparent to visible light for radiation detection purposes, due to the band gap of the material being larger than the equivalent energy for that wavelength range. Consequently it is possible that a $\mathrm{SiC}$ detectors under illumination from an optimum wavelength of light during irradiation could result in stable operation.

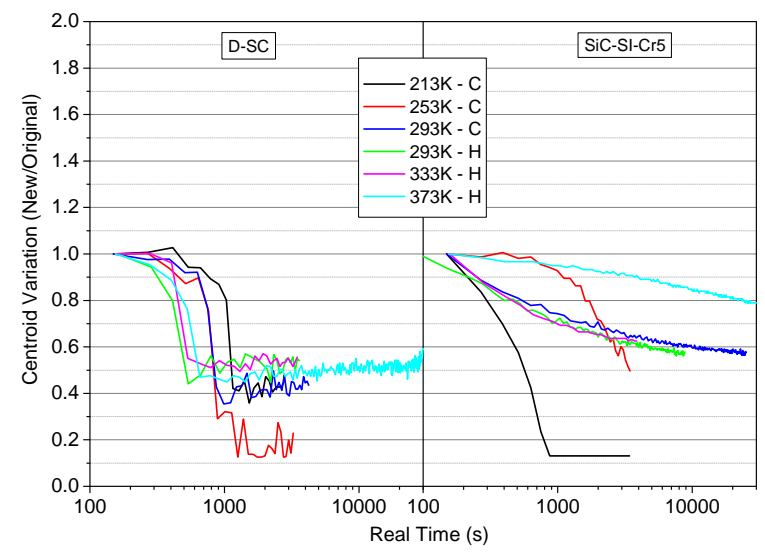

Figure 9: Variation in the alpha energy centroid peak position against irradiation time as a function of temperature for an Am-241 alpha radiation source (60kBq) with flux for $202 \alpha / \mathrm{s}$ D-SC and $282 \alpha / \mathrm{s}$ for SiC-SI. Suffix 'C' and 'H' represent the cryostat used for cooling and heating respectively. Data was taken at $293 \mathrm{~K}$ in both cryostats for comparison. All testing was conducted at $6.4 \times 10^{-1} \mathrm{mbar}$. 
The effect of temperature on the alpha polarisation rate was also investigated, the results of which are presented in Figure 9. For D-SC polarisation begins to affect the centroid peak position between 100-200s at all temperatures studied, with the rate of polarisation being a similar order of magnitude $\left(\mathrm{P}_{\max }\right.$ to $\mathrm{P}_{\min }$ is between 500-1000s). Variations between heating or cooling the material are related to slightly different test setups between the heating or cooling plate as the $293 \mathrm{~K}$ measurements vary by a similar amount.

Conversely to this, the semi-insulating $\mathrm{SiC}$ detector ( $\mathrm{SiC}-\mathrm{SI}-\mathrm{Cr} 5$ ) shows that the polarisation rate actually decreases as the temperature increases, a characteristic also demonstrated by Guerrero et al. in D [45]. Furthermore, it begins to stabilise at a $P_{\text {min }}$ above the noise threshold, allowing for long term stable operation. These results therefore suggest that the energy imparted to the detector at $373 \mathrm{~K}$ is sufficient to stimulate shallow thermal detrapping of charge carriers resulting in improved charge transport in the material due to a diluted space charge build up.

At lower temperatures the polarisation rate actually increases relative to at $293 \mathrm{~K}$, with a rapid polarisation to the noise threshold rather than a steady asymptotic decline, suggesting trapping of thermally generated charge carriers is an important mechanism for polarisation in these detectors. Although it is worth noting that the period prior to polarisation taking effect is increased at $253 \mathrm{~K}$. It is unlikely that test setup variations between heating and cooling resulted in these different observations as the $293 \mathrm{~K}$ observations were similar.

These are extremely interesting observations as they suggest semi-insulating $\mathrm{SiC}$ can be a stable radiation detector at higher temperatures with the detection capabilities being maintained, as has been shown in [6]. Unlike other detectors where cooling is necessary (CdTe with Peltier cooling, Ge with liquid nitrogen), heating a detector is relatively simple and low cost (i.e. heating element) [53]. In fact, many of the proposed applications for this material are harsh environments at high temperatures (nuclear reactor controls, fusion, oil and gas welling etc) [6][54], meaning the application itself could be sufficient to stabilise the detector.

\section{Conclusion}

The alpha irradiation induced polarisation phenomena in semi-insulating silicon carbide (SiC-SI) has been thoroughly characterised and compared to well known, wide band gap semiconductor materials, single crystal diamond 
(D-SC) and polycrystalline diamond (D-PC). The polarisation rate as an effect of incident flux, bias and temperature was determined, with the depolarisation rate as a function of ambient light and bias also demonstrated. Consequently it has been shown that semi-insulating silicon carbide can be stably operated as radiation detectors.

The observed polarisation effect within the SiC-SI materials studied were similar to the effects found with polycrystalline diamond such that polarisation effect was paralyzable and non-recoverable without external interference.

The investigation showed that the polarisation rate increases with incident alpha flux and low electric field, however with certain conditions (incident radiation flux) and setup (bias) stable detector operation can be maintained.

It has also been shown that the a polarised detector can be reset to its preirradiation characteristics (depolarised) through exposure to ambient light. Furthermore, the detector can be depolarised when an unirradiated detector is held at $0 \mathrm{~V}$, demonstrating that the polarisation effect is a result of shallow charge carrier trapping.

Furthermore, recovery can be achieved within 1min using both $0 \mathrm{~V}$ bias and ambient light together, where as the performance is actually improved for future irradiations (i.e. lower polarisation rate) when the material is illuminated while under bias. Therefore a stable detector could be possible in applications where a light source illuminates the material.

Finally, it has been shown that the the polarisation rate can be significantly reduced and stable operation maintained when the temperature is increased to $373 \mathrm{~K}$. In fact an increase in polarisation rate was observed at lower temperatures $(253 \mathrm{~K}, 213 \mathrm{~K})$. This finding is extremely important as the use of $\mathrm{SiC}$ is often discussed for harsh environmental conditions with increased temperatures.

\section{Acknowledgements}

The authors would like to acknowledge the staff at the University of Surrey for there assistance with testing and analysis. The authors would also like to thank Loadpoint for their work during the detector fabrication process.

This work was funded by the United Kingdom Science Technology Funding Council (STFC grant number: ST/H003959/1) and in collaboration with AWE. 
[1] K. C. Mandal, R. M. Krishna, P. G. Muzykov, S. Das, T. S. Sudarshan, Characterization of Semi-Insulating 4h Silicon Carbide for Radiation Detectors, IEEE Transactions on Nuclear Science 58 (4) (2011) 19921999. doi:10.1109/TNS.2011.2152857.

[2] F. Nava, P. Vanni, M. Bruzzi, S. Lagomarsino, S. Sciortino, G. Wagner, C. Lanzieri, Minimum Ionizing and Alpha Particles Detectors Based on Epitaxial Semiconductor Silicon Carbide, IEEE Transactions on Nuclear Science 51 (1) (2004) 238-244. doi:10.1109/TNS.2004.825095.

[3] G. Bertuccio, R. Casiraghi, A. Cetronio, C. Lanzieri, F. Nava, Silicon carbide for high resolution X-ray detectors operating up to $100^{\circ} \mathrm{C}, \mathrm{Nu}-$ clear Instruments and Methods in Physics Research Section A: Accelerators, Spectrometers, Detectors and Associated Equipment 522 (3) (2004) 413-419. doi:10.1016/j.nima.2003.11.413.

[4] F. Ruddy, J. Seidel, H. Chen, A. Dulloo, S.-H. Ryu, High-resolution alpha-particle spectrometry using $4 \mathrm{~h}$ silicon carbide semiconductor detectors, IEEE Transactions on Nuclear Science 53 (3) (2006) 1713-1718. doi:10.1109/TNS.2006.875155.

[5] R. Flammang, J. Seidel, F. Ruddy, Fast neutron detection with silicon carbide semiconductor radiation detectors, Nuclear Instruments and Methods in Physics Research Section A: Accelerators, Spectrometers, Detectors and Associated Equipment 579 (1) (2007) 177-179.

[6] M. Hodgson, A. Lohstroh, P. Sellin, D. Thomas, Characterisation of Silicon Carbide and Diamond Detectors for Neutron Applications., Measurement Science and TechnologyIn Review.

[7] M. Rogalla, K. Runge, A. Söldner-Rembold, Particle detectors based on semi-insulating silicon carbide, Nuclear Physics B-Proceedings Supplements 78 (1) (1999) 516-520.

[8] G. Bertuccio, D. Puglisi, A. Pullia, C. Lanzieri, X-gamma Ray Spectroscopy With Semi-Insulating 4h-Silicon Carbide, IEEE Transactions on Nuclear Science 60 (2) (2013) 1436-1441. doi:10.1109/TNS.2013.2252019. 
[9] M. Hodgson, A. Lohstroh, P. Sellin, D. Thomas, Neutron detection performance of silicon carbide and diamond detectors with incomplete charge collection properties, Nuclear Instruments and Methods in Physics Research Section A: Accelerators, Spectrometers, Detectors and Associated Equipmentdoi:10.1016/j.nima.2016.11.006.

[10] F. H. Ruddy, J. G. Seidel, R. W. Flammang, R. Singh, J. Schroeder, Development of radiation detectors based on semi-insulating silicon carbide, in: IEEE Nuclear Science Symposium Conference Record. NSS '08, 2008, pp. 449 -455. doi:10.1109/NSSMIC.2008.4775205.

[11] A. Lohstroh, P. J. Sellin, S. G. Wang, A. W. Davies, J. M. Parkin, Mapping of polarization and detrapping effects in synthetic single crystal chemical vapor deposited diamond by ion beam induced charge imaging, Journal of Applied Physics 101 (6) (2007) 063711.

[12] L. Lan, O. Xiaoping, T. Xinjian, X. Liangbin, C. Na, L. Bing, Z. Xiaodong, Priming effect on a polycrystalline CVD diamond detector under 60-Co $\gamma$-rays irradiation, Nuclear Instruments and Methods in Physics Research Section A: Accelerators, Spectrometers, Detectors and Associated Equipment 672 (2012) 29-32. doi:10.1016/j.nima.2011.12.022.

[13] P. Bergonzo, D. Tromson, C. Mer, B. Guizard, F. Foulon, A. Brambilla, Particle and Radiation Detectors Based on Diamond, physica status solidi (a) 185 (1) (2001) 167-181. doi:10.1002/1521396X(200105)185:1¡167::AID-PSSA167¿3.0.CO;2-F.

[14] M. Guerrero, D. Tromson, M. Rebisz, C. Mer, B. Bazin, P. Bergonzo, Requirements for synthetic diamond devices for radiotherapy dosimetry applications, Diamond and Related Materials 13 (11-12) (2004) 20462051. doi:10.1016/j.diamond.2004.07.026.

[15] G. F. Knoll, Radiation detection and measurement, 4th Edition, John Wiley, Hoboken, N.J, 2010, ISBN 97804701314800470131489.

[16] C. R. Leão, V. Lordi, Ionic current and polarization effect in TlBr, Physical Review B 87 (8) (2013) 081202. doi:10.1103/PhysRevB.87.081202. 
[17] B. Dönmez, C. L. Thrall, Z. He, L. J. Cirignano, H. Kim, K. S. Shah, Investigation of polarization effect with $\mathrm{TlBr}$ detectors at different operating temperatures, in: Nuclear Science Symposium Conference Record (NSS/MIC), IEEE, 2010, pp. 3773-3775.

[18] G. Lutz, Semiconductor radiation detectors device physics, Springer, Berlin; New York, 2007, ISBN 9783540716792.

[19] M. Bruzzi, D. Menichelli, S. Sciortino, L. Lombardi, Deep levels and trapping mechanisms in chemical vapor deposited diamond, Journal of Applied Physics 91 (9) (2002) 5765. doi:10.1063/1.1461891.

[20] P. Gonon, S. Prawer, D. Jamieson, Thermally stimulated currents in polycrystalline diamond films: Application to radiation dosimetry, Applied Physics Letters 70 (22) (1997) 2996. doi:10.1063/1.118768.

[21] A. A. Lebedev, Deep level centers in silicon carbide: A review, Semiconductors 33 (2) (1999) 107 to 130.

[22] A. Chynoweth, Behavior of Space Charge in Diamond Crystal Counters under Illumination. I, Physical Review 83 (2) (1951) 254.

[23] A. M. Ivanov, A. A. Lebedev, N. B. Strokan, Effect of extreme radiation fluences on parameters of $\mathrm{SiC}$ nuclear particle detectors, Semiconductors 40 (10) (2006) 1227-1231. doi:10.1134/S1063782606100186.

[24] G. S. Camarda, A. E. Bolotnikov, Y. Cui, A. Hossain, S. A. Awadalla, J. Mackenzie, H. Chen, R. B. James, Polarization Studies of CdZnTe Detectors Using Synchrotron X-Ray Radiation, IEEE Transactions on Nuclear Science 55 (6) (2008) 3725-3730. doi:10.1109/TNS.2008.2004707.

[25] P. Siffert, J. Berger, C. Scharager, A. Cornet, R. Stuck, R. Bell, H. Serreze, F. Wald, Polarization in cadmium telluride nuclear radiation detectors, Nuclear Science, IEEE Transactions on 23 (1) (1976) 159-170.

[26] C.-M. Zetterling, Institution of Electrical Engineers., Process technology for silicon carbide devices, INSPEC, London, 2002, ISBN 0852969988 9780852969984 .

[27] J. W. Glesener, Photoinduced current transient spectroscopy of boron doped diamond, Applied Physics Letters 63 (6) (1993) 767. doi:10.1063/1.109902. 
[28] S. G. Wang, P. Sellin, A. Lohstroh, Temperature-dependent hole detrapping for unprimed polycrystalline chemical vapor deposited diamond, Applied Physics Letters 88 (2) (2006) 023501.

[29] P. Sellin, J. Vaitkus, New materials for radiation hard semiconductor dectectors, Nuclear Instruments and Methods in Physics Research Section A: Accelerators, Spectrometers, Detectors and Associated Equipment 557 (2) (2006) 479-489. doi:10.1016/j.nima.2005.10.128.

[30] F. H. Ruddy, R. W. Flammang, J. G. Seidel, Low-background detection of fission neutrons produced by pulsed neutron interrogation, Nuclear Instruments and Methods in Physics Research A 598 (2009) 518-525.

[31] F. Franceschini, F. Ruddy, Silicon Carbide Neutron Detectors, in: R. Gerhardt (Ed.), Properties and Applications of Silicon Carbide, InTech, 2011.

[32] R. Bell, G. Entine, H. Serreze, Time-Dependent Polarization of CdTe Gamma-Ray Detectors, Nuclear Instruments \& Methods 117 (1) (1974) 267-271. doi:10.1016/0029-554X(74)90408-X.

[33] S. F. Kozlov, R. Stuck, M. Hage-Ali, P. Siffert, Preparation and characteristics of natural diamond nuclear radiation detectors, Nuclear Science, IEEE Transactions on 22 (1) (1975) 160-170.

[34] H. Malm, M. Martini, Polarization phenomena in CdTe nuclear radiation detectors, Nuclear Science, IEEE Transactions on 21 (1) (1974) 322-330.

[35] C. Hordequin, D. Tromson, A. Brambilla, P. Bergonzo, F. Foulon, Strong impact of x-ray radiation associated with electron beam metallization of diamond devices, Journal of Applied Physics 90 (5) (2001) 2533-2537. doi:10.1063/1.1338515.

[36] K. McKay, Electron Bombardment Conductivity in Diamond. Part II, Physical Review 77 (6) (1950) 816.

[37] A. Muraro, L. Giacomelli, M. Nocente, M. Rebai, D. Rigamonti, F. Belli, P. Calvani, J. Figueiredo, M. Girolami, G. Gorini, G. Grosso, A. Murari, S. Popovichev, D. M. Trucchi, M. Tardocchi, JET Contributors, First neutron spectroscopy measurements with a pixelated 
diamond detector at JET, Review of Scientific Instruments 87 (11) (2016) 11D833. doi:10.1063/1.4961557.

URL http://scitation.aip.org/content/aip/journal/rsi/87/ $11 / 10.1063 / 1.4961557$

[38] P. Bergonzo, D. Tromson, C. Descamps, H. Hamrita, C. Mer, N. Tranchant, M. Nesladek, Improving diamond detectors: A device case, Diamond and Related Materials 16 (4-7) (2007) 1038-1043. doi:10.1016/j.diamond.2006.11.099.

[39] G. Sato, T. Fukuyama, S. Watanabe, H. Ikeda, M. Ohta, S. Ishikawa, T. Takahashi, H. Shiraki, R. Ohno, Study of polarization phenomena in Schottky CdTe diodes using infrared light illumination, Nuclear Instruments and Methods in Physics Research A 652 (2011) 149-152.

[40] S. Mersi, E. Borchi, M. Bruzzi, R. D'Alessandro, S. Lagomarsino, S. Sciortino, A study of charge collection processes on polycrystalline diamond detectors, Nuclear Instruments and Methods in Physics Research Section A: Accelerators, Spectrometers, Detectors and Associated Equipment 530 (1-2) (2004) 146-151. doi:10.1016/j.nima.2004.05.063.

[41] C. Manfredotti, E. Vittone, C. Paolini, P. Olivero, A. Lo Giudice, Blue light sensitization of CVD diamond detectors, Diamond and Related Materials 12 (3-7) (2003) 662-666. doi:10.1016/S0925-9635(02)00327-8.

[42] M. Marinelli, E. Milani, A. Paoletti, G. Pucella, A. Tucciarone, G. Verona Rinati, M. Angelone, M. Pillon, Analysis of traps in high quality CVD diamond films through the temperature dependence of carrier dynamics, Diamond and Related Materials 12 (10-11) (2003) 1733-1737. doi:10.1016/S0925-9635(03)00204-8.

[43] A. J. Whitehead, R. Airey, C. M. Buttar, J. Conway, G. Hill, S. Ramkumar, G. A. Scarsbrook, R. S. Sussmann, S. Walker, CVD diamond for medical dosimetry applications, Nuclear Instruments and Methods in Physics Research Section A: Accelerators, Spectrometers, Detectors and Associated Equipment 460 (1) (2001) 20-26.

[44] A. Cola, I. Farella, The polarization mechanism in CdTe Schottky detectors, Applied Physics Letters 94 (10) (2009) 102113. doi:10.1063/1.3099051. 
[45] M. Guerrero, D. Tromson, C. Descamps, P. Bergonzo, Recent improvements on the use of CVD diamond ionisation chambers for radiotherapy applications, Diamond and Related Materials 15 (4-8) (2006) 811-814. doi:10.1016/j.diamond.2005.12.056.

[46] M. A. E. Abdel-Rahman, A. Lohstroh, P. J. Sellin, The effect of annealing on the X-ray induced photocurrent characteristics of CVD diamond radiation detectors with different electrical contacts, Physica Status Solidi a-Applications and Materials Science 208 (9) (2011) 2079-2086.

[47] F. Schirru, B. S. N. Singh, L. Scruton, M. A. Bentley, S. P. Fox, A. Lohstroh, P. J. Sellin, A. Banu, M. McCleskey, B. T. Roeder, E. Simmons, A. A. Alharbi, L. Trache, M. Freer, D. Parker, Development of large area polycrystalline diamond detectors for fast timing application of high-energy heavy-ion beams, Journal of Instrumentation 7 (05) (2012) P05005-P05005. doi:10.1088/1748-0221/7/05/P05005.

[48] K. Siegbahn (Ed.), Alpha-, beta- and gamma-ray spectroscopy, NorthHolland Publ. [u.a.], Amsterdam, 1965, ISBN 0444106952.

[49] E.-K. Souw, R. Meilunas, Response of CVD diamond detectors to alpha radiation, Nuclear Instruments and Methods in Physics Research Section A: Accelerators, Spectrometers, Detectors and Associated Equipment 400 (1) (1997) 69-86. doi:10.1016/S0168-9002(97)00963-7.

[50] J. Ziegler, SRIM \& TRIM, accessed 07-07-2015 (2013).

URL http://www.srim.org/

[51] P. Bergonzo, A. Brambilla, D. Tromson, C. Mer, B. Guizard, R. Marshall, F. Foulon, CVD diamond for nuclear detection applications, Nuclear Instruments and Methods in Physics Research Section A: Accelerators, Spectrometers, Detectors and Associated Equipment 476 (3) (2002) 694-700. doi:10.1016/S0168-9002(01)01668-0.

[52] P. Bergonzo, D. Tromson, C. Mer, Radiation detection devices made from CVD diamond, Semiconductor Science and Technology 18 (3) (2003) S105-S112. doi:10.1088/0268-1242/18/3/315.

[53] M. J. GUERRERO, P. Bergonzo, D. Tromson, Detecting device based on a synthetic diamond (WO2006024661 A1). 
[54] Y. M. Abubakar, A. Lohstroh, P. J. Sellin, Stability of Silicon Carbide Particle Detector Performance at Elevated Temperatures, IEEE Transactions on Nuclear Science 62 (5) (2015) 2360-2366. doi:10.1109/TNS.2015.2475421. 